\title{
A SHORT PROOF OF THE FROBENIUS THEOREM
}

\author{
ALBERT T. LUNDELL
}

(Communicated by Jonathan M. Rosenberg)

ABstract. By separating the algebraic and analytic aspect of Frobenius' theorem on involutive distributions, we are able to give a simplified proof.

The Frobenius theorem may be stated as follows [B, p. 161] :

Theorem. An $r$-distribution $\Delta$ on an $m$-manifold $M$ is involutive if and only if $\Delta$ is completely integrable.

We recall that $\Delta$ is involutive if in the neighborhood of each point of $M$ there is a local basis of vector fields $\left\{X_{1}, X_{2}, \ldots, X_{r}\right\}$ such that

$$
\left[X_{i}, X_{j}\right]=\sum_{k=1}^{r} c_{i, j}^{k} X_{k} \quad \text { for } 1 \leq i, j \leq r,
$$

and $\Delta$ is completely integrable if for each point $p \in M$ there is a coordinate system $u:(U, p) \rightarrow\left(\mathbb{R}^{m}, 0\right)$ such that $\Delta$ is spanned by $\left\{\partial / \partial u^{1}, \ldots, \partial / \partial u^{r}\right\}$.

Clearly, if $\Delta$ is completely integrable then $\Delta$ is involutive. Thus we assume that $\Delta$ is involutive.

The following proof separates the algebraic and analytic aspects of the theorem. The algebraic part is completely elementary; we give a reference for the analytic part.

Lemma 1. There is a coordinate system $v:(V, p) \rightarrow\left(\mathbb{R}^{m}, 0\right)$ such that $\Delta$ has a local basis $\left\{X_{1}, \ldots, X_{r}\right\}$ of the form

$$
X_{i}=\frac{\partial}{\partial v^{i}}+\sum_{j=r+1}^{m} b_{i}^{j} \frac{\partial}{\partial v^{j}} \quad \text { for } 1 \leq i \leq r .
$$

Proof. Choose coordinates $v:\left(V^{\prime}, p\right) \rightarrow\left(\mathbb{R}^{m}, 0\right)$ and let $\left\{Y_{1}, \ldots, Y_{r}\right\}$ be a local basis of vector fields for $\Delta$ in $V^{\prime}$. Then

$$
Y_{i}=\sum_{j=1}^{m} a_{i}^{j} \frac{\partial}{\partial v^{j}} \quad \text { for } 1 \leq i \leq r,
$$

where the functions $a_{i}^{j}$ are $C^{\infty}$ on $V^{\prime}$. Since the $Y_{i}$ are independent, the $r \times m$ matrix $A=\left(a_{i}^{j}\right)$ is of rank $r$, and without loss of generality, we may assume

Received by the editors April 29, 1991.

1991 Mathematics Subject Classification. Primary 53C99. 
that in a (possibly smaller) neighborhood $V$ of $p$ the $r \times r$ submatrix $A^{\prime}=$ $\left(a_{i}^{j}\right), 1 \leq i, j \leq r$, is nonsingular. If $A^{\prime-1}=\left(\hat{a}_{i}^{j}\right)$, we set $X_{i}=\sum_{j=1}^{r} \hat{a}_{i}^{j} Y_{j}$.

Corollary. If the r-distribution $\Delta$ is involutive, then the local basis of the lemma satisfies $\left[X_{i}, X_{j}\right]=0$ for $1 \leq i, j \leq r$.

Proof. Observe that

$$
\left[X_{i}, X_{j}\right] \in \operatorname{span}\left\{\frac{\partial}{\partial v^{r+1}}, \ldots, \frac{\partial}{\partial v^{m}}\right\} \text { for } 1 \leq i, j \leq r .
$$

On the other hand, since $\Delta$ is involutive, $\left[X_{i}, X_{j}\right] \in \operatorname{span}\left\{X_{1}, \ldots, X_{r}\right\}$ for $1 \leq i, j \leq r$. Thus

$$
\left[X_{i}, X_{j}\right] \in \operatorname{span}\left\{X_{1}, \ldots, X_{r}\right\} \cap \operatorname{span}\left\{\frac{\partial}{\partial v^{r+1}}, \ldots, \frac{\partial}{\partial v^{m}}\right\}=\cdot\{0\} .
$$

For a proof of the theorem, we now appeal to [BC, Theorem 5, p. 20], which states that if $\left\{X_{1}, \ldots, X_{r}\right\}$ is a set of independent vector fields on an open set $V$ such that $\left[X_{i}, X_{j}\right]=0$ on $W$, then for $p \in W$ there is a coordinate system $u:(U, p) \rightarrow\left(\mathbb{R}^{m}, 0\right)$ such that $X_{i}=\partial / \partial u^{i}$ for $1 \leq i \leq r$.

For a proof of the theorem using a more elementary analytic result, we quote the familiar [W, Proposition 1.53, p. 40], which follows directly from an existence theorem in ordinary differential equations. We state this as a lemma and do not provide a proof.

Lemma 2. If $X$ is a vector field with $X(p) \neq 0$, then there is a coordinate system $u:(U, p) \rightarrow\left(\mathbb{R}^{m}, 0\right)$ such that on $U, X=\partial / \partial u^{1}$.

Of course this is the case $r=1$ of $[\mathrm{BC}]$. The following proof of the Frobenius Theorem is similar to that of [CW].

The proof is by induction on $r$. The case $r=1$ is just Lemma 2. Suppose that $r \geq 2$ and the result is true for $r-1$. Using the corollary above, we may assume that in a neighborhood of $p$ the distribution $\Delta$ has a basis $\left\{X_{1}, \ldots, X_{r}\right\}$ satisfying $\left[X_{i}, X_{j}\right]=0$. The inductive hypothesis enables us to choose coordinates $v:(V, p) \rightarrow\left(\mathbb{R}^{m}, 0\right)$ so that on $V$, we have

$$
\operatorname{span}\left\{X_{1}, \ldots, X_{r-1}\right\}=\operatorname{span}\left\{\frac{\partial}{\partial v^{1}}, \ldots, \frac{\partial}{\partial v^{r-1}}\right\} .
$$

Since the $\partial / \partial v^{i}$ and the $X_{i}$ are related by a nonsingular linear transformation and $\left[X_{i}, X_{r}\right]=0$, we see that

$$
\begin{aligned}
{\left[\frac{\partial}{\partial v^{i}}, X_{r}\right] } & \in \operatorname{span}\left\{X_{1}, \ldots, X_{r-1}\right\} \\
& =\operatorname{span}\left\{\frac{\partial}{\partial v^{1}}, \ldots, \frac{\partial}{\partial v^{r-1}}\right\} \quad \text { for } 1 \leq i \leq r-1
\end{aligned}
$$

In a neighborhood of $p$ we may write $X_{r}=\sum_{i=1}^{m} a^{i} \partial / \partial v^{i}$, and the condition on brackets yields

$$
\frac{\partial a^{i}}{\partial v^{j}}=0 \quad \text { for } r \leq i \leq m \text { and } 1 \leq j \leq r-1 .
$$


Thus for $r \leq i \leq m$, the $a^{i}$ are independent of $v^{1}, \ldots, v^{r-1}$. If we set

$$
Y=X_{r}-\sum_{i=1}^{r-1} a^{i} \frac{\partial}{\partial v^{i}}=\sum_{i=r}^{m} a^{i} \frac{\partial}{\partial v^{i}},
$$

then

$$
\operatorname{span}\left\{X_{1}, \ldots, X_{r}\right\}=\operatorname{span}\left\{\frac{\partial}{\partial v^{1}}, \ldots, \frac{\partial}{\partial v^{r-1}}, Y\right\}
$$

and $Y$ depends only on $v^{r}, \ldots, v^{m}$. Using Lemma 2, we may choose coordinates $u:(U, p) \rightarrow\left(\mathbb{R}^{m}, 0\right)$ of the form

$$
u^{i}= \begin{cases}v^{i} & \text { for } 1 \leq i \leq r-1 \\ \tilde{u}^{i}\left(v^{r}, \ldots, v^{m}\right) & \text { for } r \leq i \leq m\end{cases}
$$

so that

$$
\begin{gathered}
\frac{\partial}{\partial u^{i}}=\frac{\partial}{\partial v^{i}} \quad \text { for } 1 \leq i \leq r-1, \\
\frac{\partial}{\partial u^{r}}=\frac{\partial}{\partial v^{r}}+\sum_{k=r+1}^{m} a^{k} \frac{\partial}{\partial v^{k}}=Y .
\end{gathered}
$$

We have $\left\{\partial / \partial u^{1}, \ldots, \partial / \partial u^{r}\right\}$ as a basis for $\Delta$ on $U$.

Remark. Either of these proofs will work for the Frobenius theorem with parameters, the only changes being mention of the parameters.

\section{REFERENCES}

[ BC] R. L. Bishop and R. J. Crittenden, Geometry of manifolds, Academic Press, New York, 1964.

[B] W. M. Boothby, An introduction to differentiable manifolds and Riemannian geometry, Academic Press, Orlando, FL, 1986.

[ CW] S. S. Chern and J. G. Wolfson, A simple proof of the Frobenius theorem, Manifolds and Lie Groups, Progr. Math., vol. 14, Birkhauser, Boston, 1981, pp. 67-69.

[ W] F. W. Warner, Foundations of differentiable manifolds and Lie groups, Springer-Verlag, New York, 1971. 80309

Department of Mathematics, Box 426, University of Colorado, Boulder, Colorado

E-mail address: lundell@euclid.colorado.edu 